\title{
Study on the Analytical Method of Professional Monitoring Data of Urban Dangerous Building Safety
}

\author{
Yuanmao Lin \\ Chongqing Vocational Institute of Engineering, Chongqing, China, 402260
}

Keywords: Deformation Monitoring, Analysis of Data

\begin{abstract}
The urban economic development and the overall demand of urban planning cannot be completely dismantled for all the dangerous houses in the central area of the city. In this paper, according to the standards as " the standard of dangerous building appraisal ", "code for deformation measurement of building and structure" and "standard for appraisal of reliability of civil building" and so on, long-term deformation monitoring of buildings, which observe used, and use after treatment, and have been stopped before the overall demolition, to get the data of monitoring, then analyze and process it to get the building changes, and provide basis for decision-making for the government's disposal of dangerous building.
\end{abstract}

\section{Introduction}

As Chinese urban construction speed continues to accelerate, people's living environment has been effectively improved. City housing in the course is using some dangerous buildings. According to the "dangerous housing appraisal standard", some bearing capacity of the load-bearing structure cannot meet the normal use requirements, local dangerous situation, constitute a local dangerous room, belonging to the C-class dangerous; load bearing capacity cannot meet the normal use requirements, the overall housing situation, Building dangerous buildings, belonging to D-class dangerous room. Urban dangerous rooms should be dealt with accordingly. The main means include observation or handling of use, cessation of use and overall dismantling. Due to the fact that some cities cannot be dismantled as a whole, on the basis of observing and monitoring the whole or local structural deformation of the dangerous buildings, through the monitoring of the settlement, inclination, stress, cracks and other specialization and informationization of the housing foundation, Hidden trouble to monitor, access to monitoring data and analysis.

\section{Data Analysis Process}

The analysis of the obtained monitoring data mainly includes three processes:

First, the monitoring staff to organize the inspection data and check (including gross removal), the use of reliable monitoring data to draw a single point of deformation process line, the horizontal axis for the monitoring time, the vertical axis for the deformation (settlement, tilt, cracks, etc.), To understand the basic point of a single deformation. The collected picture information remains intact, and the deformation of each monitoring point monitored by the monitoring is summarized. Due to the real-time continuous, dynamic and automated monitoring system used in the monitoring project, the " $3 \delta$ criterion" should be adhered to to eliminate the gross error when collecting the monitoring data. At the same time, after removing the gross error, in order to ensure the continuity of the data sequence, the previous period of observation data or predictions can be used to interpolate;

Second, the monitoring staff in conjunction with the structural engineering staff, combined with the foundation of the foundation, the upper load-bearing structure of the deformation and other relevant conditions, combined with various types of monitoring data for comprehensive analysis (hereinafter referred to as "joint analysis").

(1) Joint analysis is divided into three levels: a. Housing the same plane similar monitoring content of the joint analysis; b. Housing the same plane with different types of monitoring content 
of the joint analysis; c. Housing the same elevation of different monitoring content of the joint analysis. Each level of joint analysis respectively draws the corresponding deformation process line.

(2) Draw all kinds of deformation process line, the monitoring time should be the horizontal axis, the amount of deformation for the vertical axis, the figure should include map name, legend, data units and other auxiliary information.

Finally, by the professional and technical personnel to monitor the housing of the overall deformation of the data to discuss, so as to clarify the current housing stability, safety and its deformation and development trends to determine and predict.

\section{Data Analysis Method}

There are gross errors, relative errors, and relative accuracy problems in the monitoring data collection process.

(1) Judgment and rejection of gross error: When the residual of an observation is greater than 3 times the tolerance of the instrument (the nominal accuracy of the instrument), there may be a gross error, and the observation data should be removed.

(2) Relative error analysis: check the plane relative error and elevation relative error, to see their size especially the distance between the relative point of the relative error, such as a point of the relative error is much larger than other points, that The point has a problem with the observed value. Or lack sufficient data to determine the reliable location of one of the pairs.

(3) Relative accuracy analysis: When the relative accuracy of the pair is lower than the specified value, it is necessary to check the observation between the pair of points, determine whether it can improve the relative accuracy, if necessary, need to collect more observation data.

After the monitoring data are collected, the difference between the two observations at the observation point is compared with the maximum error (twice the error). When the error value is small, the anomaly of the observation point can be obtained by using the regression equation To be analyzed.

In the test of monitoring points can be used when the comparison method, when the adjacent two phases of the elevation or coordinate adjustment of the difference $\triangle$ meet the following conditions, you can determine the stability of the point.

$$
\Delta<2 \mu_{0} \sqrt{2 Q}
$$

Where $\mu_{0}$ - error in unit weight (mm);

$Q$ - check the reciprocal of the elevation or coordinates of the point.

That is, the measured value of each measurement point and its previous round of data values, when the amount of change is greater than 2 times the error, as a deformation, take the value of the displacement change value; when the amount of change is less than 2 times the error, that The monitoring point is not deformed and the change value is the measurement error.

\section{Abnormal Data Processing Methods}

In each round of monitoring of the abnormal data should be analyzed and processed, the main situation is as follows:

(1) Automatic Monitoring: the quality of monitoring results by the observation conditions, observation time, rainfall, temperature and other factors, part of the monitoring point data in the monitoring process occasionally a large fluctuations, resulting in abnormal deformation of the abnormal changes (sudden acceleration, Back and forth fluctuations, etc.). When the problem is found, the members of the monitoring project team will first carry on the internal analysis and processing, and ensure the inspection of the monitoring system in the vicinity of the monitoring point where the measurement data is abnormal, in order to ensure the correctness of the internal solution. Whether the normal work of the instrument, such as the above steps still cannot solve the problem, according to the site climate conditions, to avoid adverse weather time, re-measurement, 
the re-monitoring data and the previous and several rounds of data mapping curve comparison, the overall deformation trend of the house will be far more than the measurement range of the data removed.

(2) Manual Monitoring and Macro Inspection Monitoring: the abnormal data, the artificial re-test, check the pile is loose and the measurement tool is deformed, and combined with the macro tour, check the spot around the visible macroscopic deformation, to ensure that Site measurement and analysis of abnormal data, each monitoring data truly reflects the crack deformation.

\section{Monitor the Contents of the Data Analysis}

Monitoring data analysis includes the following aspects:

(1) Monitoring Data Processing. Real-time monitoring system uses the monitoring method of the corresponding processing software for background processing.

(2) Monitoring Data Quality Inspection. With reference to "Engineering Survey" GB50026-2007 Article 10.10 implementation. Specifically: to monitor the stability of the network point of the test to stabilize the point as a starting point, to re-calculate the coordinates of a monitoring network or elevation. And then measure its poor, if less than $2 \sqrt{2 \mu^{2} Q}$, then that its point stability, this time should be used to measure the original coordinates or elevation as the starting point of the data.

Where: $\mu$ - errors in unit weights

$$
Q \text { - the weight coefficient }
$$

(3) Analysis of Deformation of Displacement at the Monitoring Point. First, the data processing obtains the original monitoring data (monitoring value). If the measured value minus 2 times the error is less than or equal to zero, then the displacement value is equal to zero, does not indicate its displacement azimuth. If the monitoring value minus 2 times the error is greater than zero, the monitoring value is its displacement value, indicating its displacement azimuth.

Through the comparison of the monitoring results with the previous monitoring results, the cumulative change of the monitoring data, the average change, the daily average rate of change and the monthly deformation rate are calculated. The above analysis results are used to determine the deformation of the house.

(4) Comprehensive Analysis of Multiple Monitoring Data. Based on the analysis of various monitoring data, the correlation and lagging characteristics of various deformation factors of houses are analyzed by analyzing the relative change of the same kind of monitoring data and the analysis of the correlation of different kinds of monitoring data. Based on this, through the comprehensive analysis, to determine the housing deformation trend, the deformation stage and the stability of the state, put forward early warning recommendations.

\section{Pre-Alarm Program}

Monitor the Setting of the Warning Value. According to the monitoring level, content, type, cycle classification warning, divided into prompt level, warning level, processing level. When the housing deformation to reach the "warning level", should be reminded of the relevant units of the housing situation is more serious; when the housing deformation to "treatment level", when required the relevant units to deal with the dangerous room.

Early Warning Workflow. In order to ensure the high quality of the completion of housing safety professional monitoring and early warning projects, in particular, to ensure that no omission, misstatement of disaster risk, the project team developed a scientific and rational professional monitoring and early warning work flow.

Early Warning Mode. During the period of professional monitoring, the monthly system of housing is normally carried out. When the monitoring data is abnormal, the cause is found immediately, and the anti-analysis is carried out immediately according to the monitoring data, and the result is submitted to the relevant unit immediately. When the settlement of the foundation of the 
house increases, the main body of the house is tilted seriously, the load of the load-bearing structure of the house is increased, the cracks are widened, and the local collapse of the house should be notified to the relevant departments of the local government, emergency plan. Alarm mode using telephone notification, playing gong or whistle and so on.

\section{Monitoring Results Submit}

During the monitoring period, the deformation and deformation rate should be calculated according to this time, the last observation data and the observation data, and the deformation curve and the deformation rate curve will be plotted. The monitoring situation will be monitored in the form of monitoring report Timely report to the relevant units and departments, and maintain the smooth flow of communication systems. Special emergency situations should be promptly notified by telephone, SMS and other shortcuts to the competent authorities.

The monitoring data analysis method has been used in Wulong County, Chongqing Hualong Town, Furong West Road, No. 84 County Office of dangerous building safety monitoring and other projects used to achieve a good application effect. Provide reliable data protection decision-making for the government and relevant departments to.

\section{Acknowledgements}

Fund Project: Chongqing Engineering Vocational and Technical College level, number: KJB17

\section{References}

[1] Code for engineering survey GB 50026-2007.

[2] Tao Ben algae. "Statistical analysis of measurement data" [M] .. Surveying and Mapping Press, 1992

[3] Wu Zian. "Building deformation monitoring data processing" [M]. Surveying and Mapping Press. (1-4), (112-141)

[4] Huang Shengxian, Yin Hui, Jiang Zheng. "Deformation monitoring data processing" [M]. Wuhan University Press. (98-100)

[5] JGJ 125-2016 "Hazardous Housing Identification Criteria" 